\title{
A Novel Channel Estimation Method for Power Line Communications
}

\author{
Mohammad Asadpour \\ Department of electrical Engineering, University of Bonab \\ Velayat Highway, Bonab 5551761167, East Azarbaijan, Iran
}

\begin{abstract}
High speed data can be transmitted through power line channel based on orthogonal frequency division multiplexing scheme. Power line is a multipath fading and frequency selective channel which has been contaminated by impulsive noise. As a result, these deficiencies cause more bit errors and influence the quality of channel estimation. In this article, an efficient channel estimation method based on Bayesian learning is presented. A new kernel function as well as proper hyper-parameters in relevance vector machine is used to estimate the impulse response of power line communication channel. The bit error rate performance for hard and soft decisions is evaluated and compared. The obtained results confirm the robustness of our proposed method against to critical effects of impulsive noise and multipath as well as low computational complexity.
\end{abstract}

\section{Keywords}

Coding, impulsive noise, multipath, orthogonal frequency division multiplexing (OFDM), power line communication, relevance vector machine (RVM).

\section{INTRODUCTION}

Power line as a high speed data transmission medium, namely, Power Line Communication (PLC) is ubiquitous, in which, there is no need new communication wiring. The performance of PLC systems is affected by signal distortion due to frequency-dependent cable losses, multipath propagation and noises [1], [2]. Load variations, number of branches and wire's length in power networks change the transfer function of power line channel, randomly [3], [4]. Number of branches which form network topology, determine the number of reflections between transmitter and receiver. As a result, the power line networks are considered as a multipath propagation environment. To solve noise effects and multipath fading problems, multi-carrier Orthogonal Frequency Division Multiplexing (OFDM) as an efficient modulation technique is used to gain high data rate communications which can overcome the frequency selectivity of broadband power line [5], [6]. OFDM has high flexibility in coding, constellation and power assignment which can be managed per subcarrier [7]. Gunawan et al. have demonstrated OFDM performance in PLC systems [8]. Minimum Mean Square Error (MMSE) [9] and Maximum Likelihood (ML) [10] as traditional pilot based channel estimators minimize Least-Square (LS) errors. These techniques result to improper efficiencies where the noise is not Gaussian [11]. Efficient algorithms are needed to enhance the channel estimation performance in power line channel influenced by impulsive noise [12]-[15]. The robust channel estimation algorithms have been used in [16], [17]. In [18] an efficient channel estimation method by using a time-spread structure in OFDM has been presented. Chen et al. have applied a dual Gaussian interpolation approach based on amplitude and phase domain analysis [19]. In [20], a time synchronization technique for channel estimation has been used. Devri has employed an MLP neural network for channel estimation [21]. A channel estimation approach with impulsive noise mitigation based on compressive sensing has been considered for 1/2-rate coded-OFDM system [22]. An enhanced channel estimation method using IFFT and denoising has been presented in [23]. Huang has defined a nonlinear cost function to overcome the impulsive noise effects [24]. Channel estimation is used in discrete multi-tone communication systems in [25]. It has used relevance vector machine (RVM) with a Gaussian kernel function has been taken into consideration. In this paper, proposed RVM based algorithm along with convolutional coding is used to estimate the PLC channel. Viterbi decoding is used to decode original data. In first step, the ordinary RVM is improved by a new kernel function which has more compatible with the impulse response of PLC channel. Then the hyper-parameters' improvement based on MMSE are done to estimate the PLC channel contaminated by impulsive noise in OFDM system. Simulation results show that the proposed algorithm overcomes to recently reported power line channel estimators.

\section{SYSTEM MODEL}

OFDM as a multicarrier modulation scheme splits high data rate streams to lower data rate ones which are transmitted through narrowband flat sub-channels. Indeed, OFDM technique changes a frequency selective channel to frequency flat sub-channels by splitting the effective bandwidth to orthogonal narrow sub-bands. ISI can also be eliminated when guard interval is used. The block diagram of baseband OFDM system is shown in Figure 1.

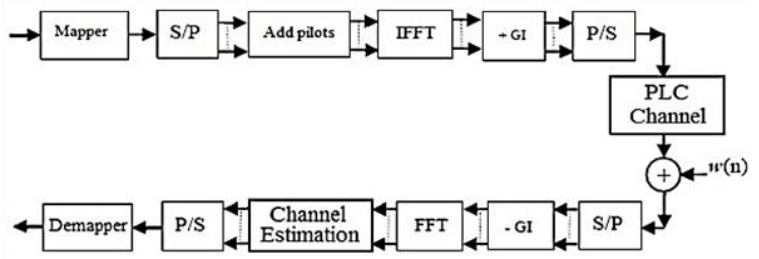

Fig 1: OFDM Block Diagram

The channel estimation based on extracted pilots is necessary at the receiver to equalize the received data. In this article, the estimation of power line communication channel contaminated by impulsive noise along with background noise is taken into consideration. Signals propagate in PLC network including direct path between transmitter and receiver as a main path and other branches connected to the system. These branches can create the reflected signals as echoes which can cause a multipath distortion. The result is considered as a 
frequency selective multipath fading model. Multipath models for power line channels have been proposed by Philips [26] and Zimmermann [27]. In this paper Zimmermann's model will be used to describe PLC channel. This model involves the superposition of $N$ different paths with weight $\mathrm{g}_{i}$ and length $\mathrm{d}_{i}$ for each path $i$. Attenuation also can be modeled by the parameters $a_{0}, a_{1}$ and $k$. Finally the multipath model for channel can be described by the following equation:

$$
H(f)=\sum_{i=1}^{N} g_{i} \cdot e^{-\left(a_{0}+a_{1} f^{k}\right) d_{i}} \cdot e^{\frac{-j 2 \pi f d_{i}}{v_{p}}}
$$

The attenuation of channel is related to first exponential function and the second one involves echo scenario. Propagation speed $v_{p}$ depends on the speed of light $c_{0}$ and dielectric constant $\varepsilon_{r}$ of the insulating material of the cable which can be calculated as:

$v_{p}=\frac{c_{0}}{\sqrt{\varepsilon_{r}}}$

Unlike usual communication channels, power lines are not Additive White Gaussian Noise (AWGN) channels. The interference due to colored broadband noise, narrowband interference and different types of impulsive disturbance is rather complicated. The result interference in PLC can be classified into five groups as colored background noise, narrowband noise, synchronous or asynchronous periodic impulsive noise with fundamental frequency (usually 50 or 60 $\mathrm{Hz}$ ) and finally asynchronous a-periodic impulsive noise[1],[28]. It can ordinarily be assumed that the first three noise classes to be stationary in a few or long period of time as seconds, minutes and sometimes even for an hour, and may be supposed as background noise. The time variant characteristics during microseconds to milliseconds can be found in two last noise classes. Switching transients anywhere in power line network cause asynchronous impulsive occurrences and more errors in data transmission. The impulses shape is often similar to superimposed damped sinusoids. The time-domain representation of two examples is shown in Figure 2. The performance of the estimation methods will be degraded by inaccurate estimation of the impulsive noise positions and therefore the optimum channel estimation will be required. LS algorithm is the simplest popular technique to estimate the channel which is usually degraded by AWGN and inter carrier interference (ICI). Bayesian theory can be used to model the relevance vector machine (RVM) technique as a linear model with the marginal and conditional Gaussian distribution. The sparse distribution on weights in a Bayesian regression model using a suitable kernel function results to sparseness. The benefits of probabilistic predictions, automatic estimation of 'nuisance' parameters and the facility to use any basis functions are the advantages accounted for RVM. Usually, RVM predictions are modeled based on a function as $f(x)$ which can be defined over the input space [29]. Based on a linear combination of $M$ basic kernel functions as: $\varphi(x)=\left(\varphi_{1}(x), \varphi_{2}(x), \ldots, \varphi_{M}(x)\right), f(x)$ can be obtained as follows:

$$
f(x ; w)=\sum_{i=1}^{M} w_{i} \varphi_{i}(x)=\boldsymbol{w}^{T} \boldsymbol{\varphi}(x)
$$

Where $\boldsymbol{w}=\left(w_{1}, w_{2}, \ldots, w_{M}\right)^{\mathrm{T}}$ as a vector must be optimally estimated. In this method, learning the general models denoted by (3) is accomplished by a Bayesian probabilistic scheme. In this article, our predictions will be based on RVM model to estimate the PLC channel. A new kernel function is defined which plays an important role to get good results in channel estimation. The received complex-valued signals as an OFDM symbol will be used in RVM method to estimate the impulse response of the PLC channel in baseband model. Our proposed block diagram shown in Figure 3, will be used to estimate the multipath PLC channel based on a training sample of complex-valued functions. At first, an initial estimation of PLC channel is obtained by traditional estimators, such as LS technique, using pilot based OFDM symbol as a training data. Two parallel paths in block diagram use RVM model on real and imaginary parts of the received input data. Concurrently, optimum initial values for necessary parameters are calculated to learn the RVM technique as well as possible. At the end of estimation, two parts of estimated channel response are merged to form the total and more actual complex-valued channel impulse response. In our method, a pseudo-random sequence as pilots, $X_{\mathrm{p}}(n)$, (with $\left|X_{\mathrm{p}}(n)\right|=\mathrm{k}$; for $n=0, \ldots, N-1)$, is applied. $\mathrm{k}$ is an adjustable amplitude of pilots which can control the local signal to noise ratio in the subcarrier locations and can save the transmitted power.
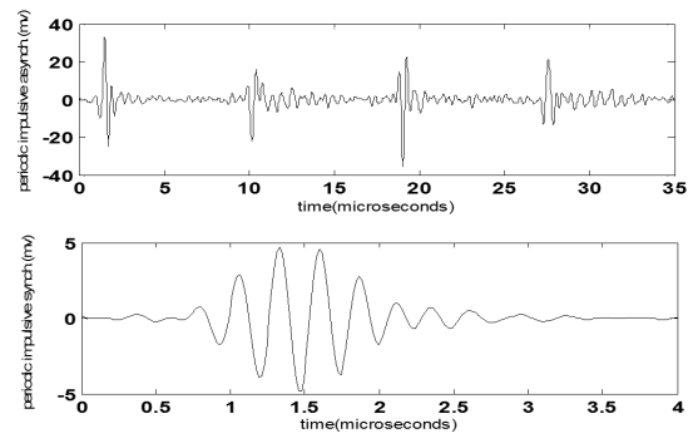

Fig 2: Impulsive events in time-domain

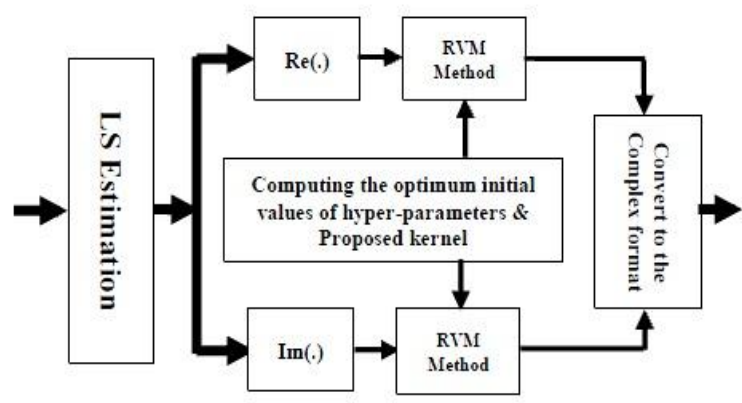

Fig 3: Proposed block diagram

The pilot symbols in receiver can be written as:

$$
\mathbf{R}_{\mathrm{p}}=\mathbf{X}_{\mathrm{p}} \mathbf{H}_{\mathrm{p}}+\mathbf{N}_{\mathrm{p}}
$$

Where $\mathbf{R}_{\mathbf{p}}$ is the $N \times 1$ received pilot signal, $\mathbf{X}_{\mathbf{p}}$ is the diagonal matrix of transmitted pilots, $\mathbf{N}_{\mathbf{p}}$ is additive noise including the AWGN and impulsive noises in all pilot locations and $\mathbf{H}_{\mathbf{p}}$ is pilot positioned frequency response of the channel. The estimation of channel frequency response in all subcarrier locations both pilot and data is main objective. It can be 
denoted by $\mathrm{H}_{m},(m=0,1,2, \ldots, N-1)$ as the FFT of $\mathrm{L}$ unknown time samples, where $\mathrm{L}$ based on maximum delay spread is not further than the equivalent length of guard interval. The initial channel estimation can be achieved by using (4) as follows [25]:

$$
\begin{aligned}
\tilde{\mathbf{H}}_{\mathrm{p}} & =\mathbf{X}_{\mathrm{p}}^{\mathrm{H}} \mathbf{R}_{\mathrm{p}}=\mathbf{X}_{\mathrm{p}}^{\mathrm{H}} \mathbf{X}_{\mathrm{p}} \mathbf{H}_{\mathrm{p}}+\mathbf{X}_{\mathrm{p}}^{\mathrm{H}} \mathbf{N}_{\mathrm{p}} \\
& =k \mathbf{H}_{\mathrm{p}}+\mathbf{N}_{\mathrm{p}}^{\prime}
\end{aligned}
$$

Where $(.)^{\mathbf{H}}$ notation indicates the Hermitian-symmetric property. Also ' $k$ ' can control the signal to noise ratio in any pilot locations in frequency domain. If the channel is assumed to be noise free then $\tilde{\mathbf{H}}_{\mathbf{p}}$ can give the actual frequency response of the channel. In practice, however, $\tilde{\mathbf{H}}_{\mathbf{p}}$ includes the channel which are degraded by the additive noises, $\mathbf{N}_{\mathbf{p}}^{\prime}$. This is simply LS estimation and the following result can be obtained:

$$
\mathbf{H}_{\mathrm{LS}}=\frac{1}{\mathrm{k}} \mathbf{X}_{\mathrm{p}}^{\mathrm{H}} \mathbf{R}_{\mathrm{p}}=\frac{1}{\mathrm{k}} \tilde{\mathbf{H}}_{\mathrm{p}}
$$

At the next step, IFFT of (5) results to the following equation in time domain:

$$
\tilde{\mathbf{h}}=\mathrm{kh}+\mathbf{n}^{\prime}
$$

Where $\tilde{\boldsymbol{h}}=\left[\tilde{h}_{0}, \tilde{h}_{1}, \ldots, \tilde{h}_{N-1}\right]^{T}$, is the observation vector, $\boldsymbol{h}=\left[h_{0}, h_{1}, \ldots, h_{v}, 0, \ldots, 0\right]^{T}$ is the actual channel impulse response, and $\boldsymbol{n}^{\prime}=\left[n_{0}^{\prime}, n_{1}^{\prime}, \ldots, n_{N-1}^{\prime}\right]^{T}$ denotes additive noise vector with variance $\sigma^{\prime 2}$. Now, RVM algorithm based on sparse Bayesian regression model is applied to estimate $\boldsymbol{h}$ from the observations, $\tilde{\boldsymbol{h}}$. It sets a few regression weights to zero and as a consequence, the noise fitting in $\tilde{h}$ is canceled. As in (3), the channel can be approximated using the function $f$ which is the linear combination of kernel functions as:

$$
f(n)=\sum_{i} w_{i} \varphi(n-i)
$$

This equation is the convolution of regression weight vector and kernel function which can be written in matrix form as follows:

$$
f=\phi w
$$

Where $\phi_{\mathrm{ij}}=\varphi(i-j)$ is $(i, j)^{\text {th }}$ element of $v \times v$ kernel matrix and $\boldsymbol{w}$ is a column weights vector which includes $v$ entries. In most researches, kernel $\phi(n)$ has been considered as a Gaussian function but in this paper a new kernel function with low complexity is introduced which has more correlative characteristics to PLC channel impulse response so that the best fitting is obtained. A number of various impulse responses used in most references based on Zimmerman model [27] are shown in Figure 4. It is clear that the suitable basic function as kernel in our proposed method is more compatible with triangle model. As a result, the following basic kernel as a shifted triangle function is proposed:

$$
\phi(n)=A \times\left|n+\frac{v}{4}\right|
$$

Where $A$ is amplitude of proposed kernel and $v$ is cyclic prefix size which is needed to fit as much as possible in learning phase. In addition to proper fitting of this kernel with channel impulse response, it's complexity with respect to Gaussian kernel function in conventional RVM is significantly low.

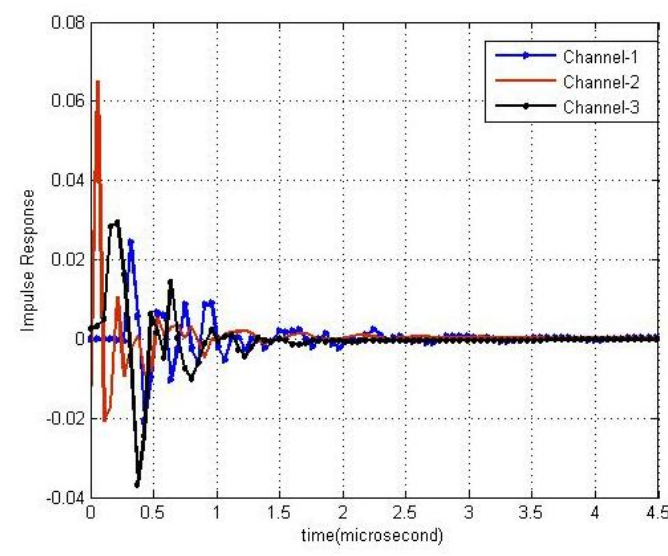

Fig 4: Three types of channel impulse response

Now applying the RVM method to the initial estimation of the channel as (7) can give the data in two classes as: approximately actual channel and noise which can be modeled as following equation.

$$
\tilde{h}=f+e=\phi w+e
$$

Where $f=\left[f_{0}, f_{1}, \ldots, f_{v}\right]^{T}$ is the approximation function and $\boldsymbol{e}=\left[e_{0}, e_{1}, \ldots, e_{\mathrm{v}}\right]^{T}$ denotes the error vector in regression model. The errors are assumed independent Gaussian random variables, with variance $\boldsymbol{\sigma}^{\mathbf{2}}$ and zero mean which are identically distributed as follows:

$$
p(\boldsymbol{e})=\prod_{i=1}^{\nu} N\left(e_{i} \mid 0, \boldsymbol{\sigma}^{2}\right)
$$

If a flexible Gaussian prior over the weights $\boldsymbol{w}$ and Bayesian inference is used, (12) with individual hyper-parameter for each weight can be written as [29], [30]:

$$
p(\boldsymbol{w}, \boldsymbol{\alpha})=\prod_{i=1}^{\nu} N\left(w_{i} \mid 0, a_{i}^{-1}\right)
$$

The posterior over the weights is then obtained from Bayesian rule:

$$
p\left(\boldsymbol{w} \mid \tilde{\boldsymbol{h}}, \boldsymbol{\alpha}, \boldsymbol{\sigma}^{2}\right)=\mathrm{N}(\boldsymbol{w} \mid \boldsymbol{\mu}, \Sigma)
$$

With

$$
\begin{aligned}
& \sum=\left(\phi^{T} B \boldsymbol{\phi}+\boldsymbol{A}\right)^{-1} \\
& \boldsymbol{\mu}=\sum \boldsymbol{\phi}^{T} \boldsymbol{B} \tilde{\boldsymbol{h}}
\end{aligned}
$$

Where $\mathbf{B}=\sigma^{2} \mathbf{I}_{v}, \mathbf{A}=\operatorname{diag}\left(\alpha_{0}, \alpha_{1}, \ldots, \alpha_{v}\right)$ and $\mathbf{I}_{v}$ is the $v \times v$ 
identity matrix. By integrating out the weights, the marginal likelihood for $\alpha, \sigma^{2}$ is obtained:

$$
p\left(\tilde{\boldsymbol{h}} / \alpha, \sigma^{2}\right)=N\left(\tilde{\boldsymbol{h}} / 0,\left(B^{-1}+\phi A^{-1} \phi^{T}\right)\right)
$$

The result regression estimation is given by $\boldsymbol{h}_{\mathrm{RVM}}=\boldsymbol{\phi} \boldsymbol{\mu}$ where $\boldsymbol{\alpha}$ and $\sigma$ can be computed by maximizing the conditional probability as $p\left(\boldsymbol{\alpha}, \sigma^{2} / \tilde{\boldsymbol{h}}\right)$. The maximum likelihood of (17) is corresponding to the maximum of $p\left(\boldsymbol{\alpha}, \sigma^{2} / \tilde{\boldsymbol{h}}\right)$ when hyper-prior is uniform assumed [29]. The maximum value of (17) respected to $\alpha$ and $\sigma^{2}$ can be obtained as follows [31]:

$$
\alpha_{i}^{\text {new }}=\frac{\gamma_{i}}{\mu_{i}^{2}}
$$

and

$$
\sigma_{\text {new }}^{2}=\frac{\|\boldsymbol{t}-\boldsymbol{\phi} \boldsymbol{\mu}\|^{2}}{v-\sum_{i} \gamma_{i}}
$$

Where $\mu_{\mathrm{i}}$ is the i'th weight of posterior mean given by (16) and $\gamma_{i}=1-\alpha_{i} \sum_{i i}$. The $\sum_{i i}$ is the i'th diagonal element of the posterior weight covariance matrix based on current $\boldsymbol{\alpha}$ and $\sigma$ values. In this algorithm, the initial values of the hyperparameters for convergence of the learning process and proper performance are very important. $\boldsymbol{\alpha}$ and $\sigma$ based on MMSE criterion are jointly determined. This is similar to Expectation-Maximization (EM) algorithm [31] that proceeds by repeating of the following steps:
1) Calculate the posterior weight covariance matrix, $\Sigma$
2) Find the posterior mean weight, $\boldsymbol{\mu}$
3) Update the hyper-parameters, $\boldsymbol{\alpha}$ and $\sigma$
4) Calculate the impulse response of estimated channel as: $\boldsymbol{h}_{\text {estimated }}=\boldsymbol{\phi} \boldsymbol{\mu}$

\section{SIMULATION RESULTS}

To evaluate the performance of the proposed method applied to PLC channels, computer simulations are carried out based on parameters in Table 1. Meanwhile, SNR and SNIR parameters are used in order to compare our proposed algorithm with the other methods as conventional RVM and Huang methods. These parameters are defined as follows:

$$
S N R=\frac{P_{S}}{P_{N}}
$$

Where PS and PN are the power of transmitted signal and additive white Gaussian noise power, respectively and

$$
S N I R=\frac{P_{S}}{(1-p) P_{N}+p \cdot P_{I}}
$$

Where $P_{I}$ is the impulsive noise power and $p$ is the parameter which can control impulsive and additive white Gaussian noise effects.

In all simulations the PLC channel model is characterized based on parameters in Table 2 which is modeled by Zimmermann multipath model as (1) [27].

After proposing triangle model for kernel, it is demonstrated the effects of initial values of the hyper-parameters $\alpha$ and $\sigma$. Figure 5, shows the MSE criterion respected to the initial values of these parameters. It is shown that there are at least two local minimums and the optimum values in the steady state should be chosen in Figure 5, as: $\alpha_{i}=8 \times 10-7, \sigma=0.35$. After applying the obtained optimum values to key parameters in proposed method, simulation results for channel estimation in both time and frequency domains are shown in Figure 6, with $4 \%$ impulsive noise effect and $\mathrm{SNR}=0 \mathrm{~dB}$. In this figure, our proposed method is compared with Huang method along with actual impulse response of the PLC channel. As shown, good results are obtained respected to the Huang method.

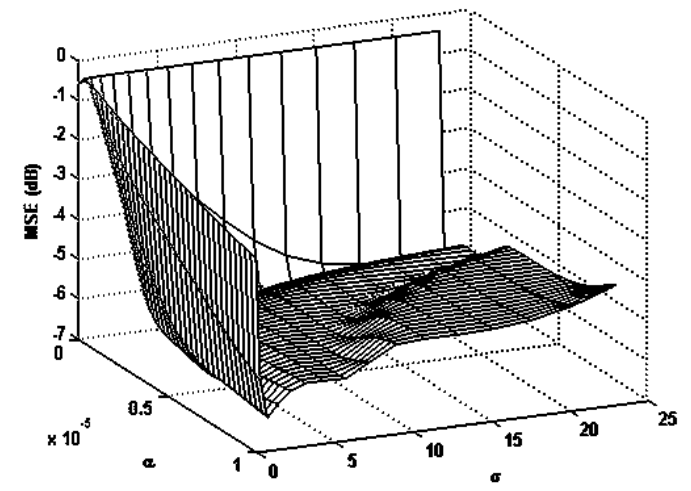

Fig 5: MSE respected to the hyper-parameters variation
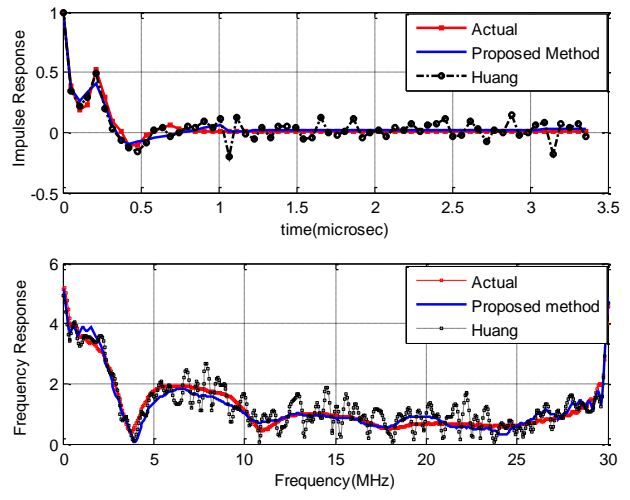

Fig 6: Impulse response curves with $\mathrm{SNR}=0 \mathrm{~dB}$, impulse noise effect $=4 \%$

Table 1. Simulation Parameters

\begin{tabular}{|c|c|}
\hline parameter & Value \\
\hline Encoder & Convolutional \\
\hline Decoder & Viterbi (Soft \& Hard Decisions) \\
\hline Number of subcarriers & $64,128,360,3072$ \\
\hline FFT size & $256,512,4096$ \\
\hline Pilot spacing & 4 \\
\hline Size of Cyclic Prefix & 64,512 \\
\hline Baseband modulation & BPSK, QPSK \\
\hline Channel type & PLC(AWGN + Impulsive noise) \\
\hline
\end{tabular}


Table 2. Parameters of four-path model

\begin{tabular}{|c|c|c|}
\hline \multicolumn{3}{|c|}{ Attenuation parameters } \\
\hline $\mathrm{K}=$ & $\mathrm{a}_{0}=0$ & $\mathrm{a}_{1}=7.8 \times 10^{-10} \mathrm{~s} / \mathrm{m}$ \\
\hline \multicolumn{3}{|c|}{ Path parameters } \\
\hline $\mathbf{i}$ & $\mathbf{g}_{\mathbf{i}}$ & $\mathbf{d}_{\mathbf{i}} / \mathbf{m}$ \\
\hline 1 & 0.64 & 200 \\
\hline 2 & 0.38 & 222.4 \\
\hline 3 & -0.15 & 244.8 \\
\hline 4 & 0.05 & 267.5 \\
\hline
\end{tabular}

In this article in order to represent more differences between our proposed method and Huang method, MSE parameter is investigated in the presence of impulsive noise effects. For instance, in Figure 7, when the effect of impulsive noise is increased, about $4 \mathrm{~dB}$ improvement in MSE for $60 \%$ impulsive noise effect in our proposed method respected to Huang method is achieved. Figure 8, shows MSE comparison of our proposed method with Huang and improved complex RVM approaches, for 3072 subcarriers and 512 cyclic prefix size according to PLC standard P1901.

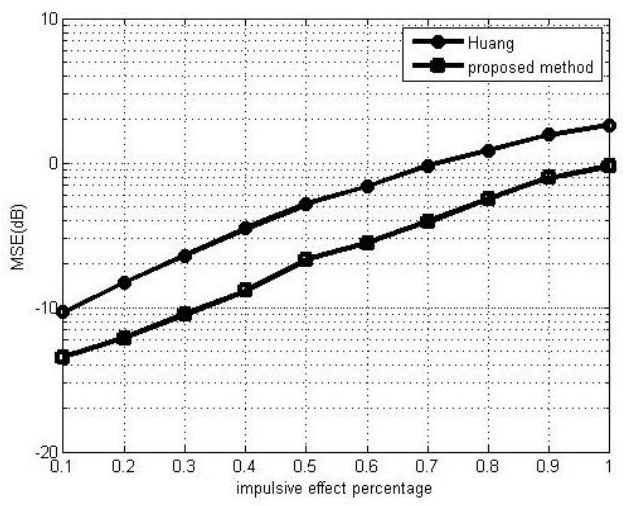

Fig 7: Impulsive noise effects, BPSK, $N=256, \mathrm{Nc}=64$, $\mathrm{CP}=64$, Pilot space $=4$

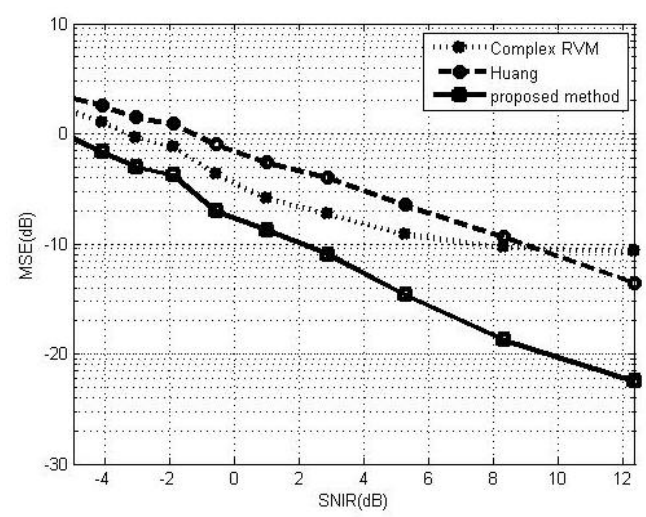

Fig 8: $\mathrm{MSE}$ comparison, $\mathrm{BPSK}, \mathrm{N}=4096, \mathrm{Nc}=3072, \mathrm{CP}$ $=512$, Pilot space $=4$

In order to improve the channel capacity, forward error correction (FEC) is needed which is done by adding some carefully designed redundant information to the data being transmitted through the channel. The process of adding this redundant information is known as channel coding. Convolutional coding is one of the two major forms of channel coding. There are a variety of useful convolutional codes and a variety of algorithms for decoding the received coded information sequences to recover the original data. Convolutional codes are usually described using two parameters: the code rate and the constraint length. The code rate, $\mathrm{k} / \mathrm{n}$, is expressed as a ratio of the number of bits into the convolutional encoder $(\mathrm{k})$ to the number of channel symbols output by the convolutional encoder ( $\mathrm{n}$ ) in a given encoder cycle. The constraint length parameter, $\mathrm{K}$, denotes the "length" of the convolutional encoder. Figure 9, shows a typical convolutional encoder diagram used in this article with code rate $1 / 2$, a constraint length of 7 , a generator polynomial matrix of [171 133] . Viterbi decoding is one of the two types of decoding algorithms used with convolutional encoding. In this paper, convolutional encoder using Viterbi decoder with hard and soft decisions is applied to our proposed algorithm. Figure 10, compares the results due to proposed algorithm with Huang method in three conditions: un-coded, hard and soft decision Viterbi decoding for 128 subcarriers and $\mathrm{CP}=64$. The convolutional encoder in these simulations has code rate $1 / 2$ and constraint length of the code 7 . The results show the appropriate improvements of proposed method respected to the Huang and any un-coded methods. For example, for $\mathrm{BER}=10^{-3}$ in soft decision decoding, about $6 \mathrm{~dB}$ and $2.5 \mathrm{~dB}$ SNIR improvements of our method respected to un-coded and Huang methods are achieved, respectively. Figure 11, shows another results for given parameters in figure. It is clear that the results related to soft decision is better than two others but the system complexity is certainly increased. There is tradeoff between complexity and estimation quality in our proposed method.

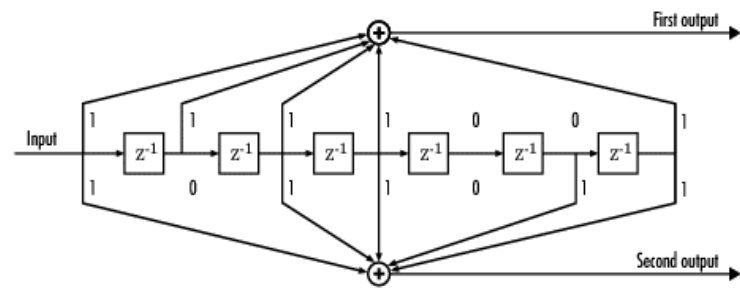

Fig 9: Convolutional Encoder diagram with rate=1/2, Constraint length $=7$

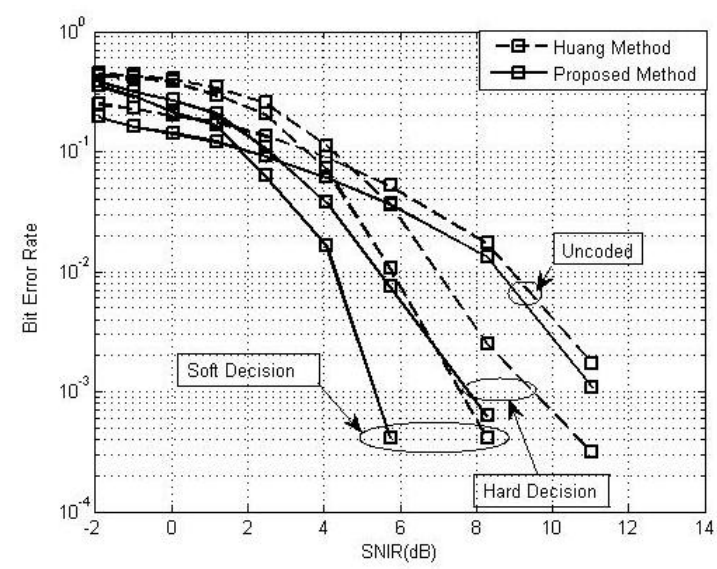

Fig 10: BER comparison, $B P S K, N=512, N c=128, C P=64$, Pilot space $=4$ 


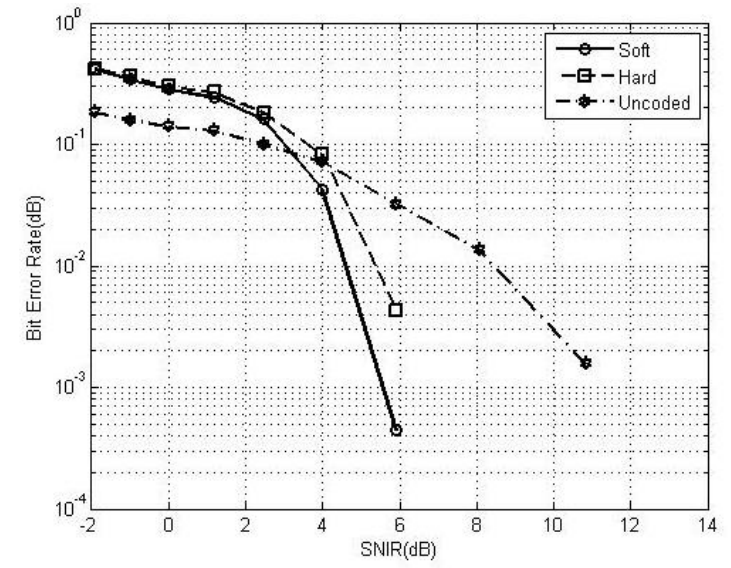

Fig 11: $B E R$ comparison, $B P S K, N=512, N c=256, C P=128$, Pilot space $=4$

\section{CONCLUSION}

The In this article, it was proposed new kernel with optimum hyper-parameters in relevance vector machine method. Also the complex-valued analysis based on RVM was used to estimate the power line communication channel. This proposed algorithm caused a good enhancement in the obtained channel estimation result. It was shown that MSE and BER parameters of our proposed method have good results in any conditions. Also the increasing of impulsive noise effect in our proposed method was compared with recently reported methods as Huang and improved conventional RVM with Gaussian kernel function. The robustness of our method against to impulsive noise effects was completely proved. Finally, in order to improve the channel capacity, convolutional coding along with Viterbi decoding as an FEC technique was applied to our algorithm. The obtained BER results confirm more considerable improvements of proposed method respected to the others.

\section{REFERENCES}

[1] M. Zimmermann and K. Dostert, "Analysis and modeling of impulsive noise in broad-band power line communications," IEEE Trans. Electromagn. Compat., vol. 44, pp. 249-258, 2002.

[2] M. Götz, M. Rapp, and K. Dostert, "Power Line Channel Characteristics and Their Effect on Communication System Design," IEEE Commun.Mag., vol. 42, pp. 7886, 2004.

[3] F. J. Canete, L. Diez, J. A. Cortes, and J. T. Entrambasaguas, "Broad-band modeling of indoor power-line channels," IEEE Trans. Consum. Electron., vol.48, pp. 175-183, 2002.

[4] P. Amirshahi and M. Kavehrad, "High-frequency characteristics of overhead multiconductor power lines for broadband communications," IEEE J. Sel. Areas Commun., vol. 24, pp. 1292-1303, 2006.

[5] L. Lampeand J. Huber, " Bandwidth efficient power line communications based on OFDM," AEU-Arch. Elektr. Ubertragungstech., vol. 54, pp. 2-12, 2000.

[6] J. Grando, A. Torralba, J. Chavez, and V. BaenaLecuyer, "Multi-carrier receiver for broadband power line communications," IEEE Trans. Consum. Electron., vol. 53, pp. 1293-1301, 2007.
[7] S. Hara and R. Prasad, "Multicarrier Techniques for 4G Mobile Communications," Inc. Norwood, MA, USA: Artech House, 2003.

[8] Y. H. Ma, P. L. So, and E. Gunawan, "Performance Analysis of OFDM Systems for Broadband power line communications under Impulsive Noise and Multipath effects," IEEE Trans. Power Del., vol. 20, pp. 674-682, 2005.

[9] O. Edfors, M. Sandel, J. J. Van de Beek, S. K. Wilson, and P. O. Borjesson, "OFDM Channel estimation by Singular value decomposition," IEEE Trans. Commun., vol. 46, pp. 931-939, 1998.

[10] R. Negi and J. Cioffi, "Pilot tone selection for channel estimation in a mobile OFDM system," IEEE Trans. Consum. Electron., vol. 44, pp. 1122-1128, 1998.

[11] A. Cichocki and S. Amari, Adaptive Blind Signal and Image Processing, New York: John Wiley \& Sons, 2003.

[12] A. Fatma, D. Pierre, and A. Florence, "Impulsive noise cancellation in multicarrier transmission," IEEE Trans. Commun., vol. 53, pp. 94-106, 2005.

[13] T. N. Zogakis, P. S. Chow, J. T. Aslanis, and J. M. Cioffi, "Impulse noise mitigation strategies for multicarrier modulation," in Proc. 1993IEEE ICC., Geneva, Switzerland, 23-26 May 1993, pp. 784-788.

[14] J. H. Stott, "Detection and removal of clipping in multicarrier receivers," European Patent Office. EP 1043874 , Oct. 2000.

[15] H. A. Suraweera, C. Chai, J. Shentu, and J. Armstrong, "Analysis of impulse noise mitigation techniques for digital television systems," in Proc. 8th Int. OFDM Workshop, Hamburg, Germany, 24-25 Sept. 2003, pp. 172-176.

[16] Y. Z. Zou, S. C. Chan, and T. S. Ng, "Least mean mestimate for robust adaptive filtering in impulse noise," IEEE Trans. Circuits-II, vol. 47, pp. 1564-1569, 2000.

[17] A. Moghaddamjoo and R. L. Kirlin, "Robust adaptive Kalman filtering with unknown inputs," IEEE Trans. Signal Process., vol. 37, pp. 1166-1175, 1989.

[18] Z. Huidog, H. Yong, Q. Shushan, and Y. Tianchun, "Design and implementation of channel estimation for low-voltage power line communication systems based on OFDM," J. Semicond., vol. 33, pp. 1-5, 2012.

[19] Y. Chen, Q. Zhang, Y. Ge, Y. Hu, J. Chen, N. Ding, and X. Zeng, "Algorithm and VLSI Architecture of Channel Estimation Impaired by Impulsive Noise in PLC," in Symp. 2013 IEEE 56th Int. Midwest Symp. on Circuits and systems (MWSCAS), Shanghai, China, 4-7 Aug. 2013, pp. 932-935.

[20] T. R. Oliveira, W. A. Finamore, and M. V. Ribeiro, "A Sounding Method based on OFDM Modulation for PLC Channel Measurement," in Symp. 2013 IEEE 17th Int. Symp. on Power Line Communications and Its Applications (ISPLC), 24-27 March 2013, pp. 185-190.

[21] D. R. Vanitha, "Channel estimation and modeling of power line communication," M.S. thesis, National Institute of Technology Rourkela-769 008, Odisha, India, 2013. 
[22] A. Mehboob, Li Zhang, J. Khangosstar, and K. Suwunnapuk, "Joint Channel and Impulsive Noise Estimation using Compressive Sensing for Powerline Communications," in Symp. 2013 IEEE 17th Int. Symp. on Power Line Communications and Its Applications (ISPLC), 24-27 March 2013, pp. 203-208.

[23] A. Chini, W. Yiyan, M. El-Tanany, and S. Mahmoud, "Filtered decision feedback channel estimation for OFDM-based DTV-terrestrial broadcasting system," IEEE Trans. Broadcast., vol. 44, pp. 2-11, 1998.

[24] J. Huang, P. Wang, and Q. Wan, "Robust approach for channel estimation in power line communication," J. Commun. Netw-S Kor., vol. 14, pp. 237-242, 2012.

[25] A. Tahat and N. Galatsanos, "Relevenace Vector Machines for Enhanced BER in DMT Based Systems," J. Electr. Comput. Eng., vol. 2010, pp. 1-8, 2010.

[26] H. Philipps, "Modelling of Powerline Communication Channels," in Proc. 3rd Int. Symp. Power-Line Commun. and its Applications, Lancaster, UK, 30 March - 1 April
1999, pp. 14-21.

[27] M. Zimmermann and K. Dostert, "A Multipath Model for the Power line Channel," IEEE Trans. Commun., vol. 50, pp. 553-559, 2002.

[28] C. Hensen and W. Schulz, "Time Dependence of the Channel Characteristics of Low Voltage Power-Lines and its Effects on Hardware implementation," AEÜ Int. J. Electron. Commun., vol. 54, pp. 23-32,2000.

[29] M. Asadpour, F. A. Ajhiri, B. M. Tazehkand and M. H. Seyedarabi, "Jointly RVM Based Channel Estimation and PAPR Reduction using Modified Tabu Search Algorithm in Power Line Communication Systems," J. Wireless Pers. Commun. vol. 84, no. 4, pp. 2757-2775, 2015.

[30] C. Bishop, Pattern Recognition and Machine Learning, New York: Springer, 2006.

[31] J. Berger, Statistical Decision Theory and Bayesian Analysis, New York: Springer, 1985. 